\title{
ALGEBRAIC PROPERTIES OF SMALL HANKEL OPERATORS ON THE HARMONIC BERGMAN SPACE
}

\author{
YONG CHEN, WEI HE AND YUNZHONG HU
}

\begin{abstract}
This paper completely characterizes the commuting problem of two small Hankel operators acting on the harmonic Bergman space with the symbols one being bounded and another being quasihomogeneous, or both being harmonic. The characterizations for semi-commuting problem and the product of two small Hankel operators being another small Hankel operator for certain class of symbols are also obtained.
\end{abstract}

\section{Introduction}

Let $\mathbb{D}$ be the open unit disk of the complex plane $\mathbb{C}$. Let $L^{p}=L^{p}(\mathbb{D}, d A)$ be the usual Lebesgue space on $\mathbb{D}$, where $1 \leq p \leq \infty$ and $d A$ is the normalized area measure. Let $b^{2}$ denote the harmonic Bergman space on $\mathbb{D}$ consisting of harmonic functions in $L^{2}$. It is well known that $b^{2}$ is a closed subspace of $L^{2}$, and hence is a Hilbert space by itself with respect to the inner product

$$
\langle\varphi, \psi\rangle=\int_{\mathbb{D}} \phi \bar{\psi} d A
$$

for functions $\varphi, \psi \in L^{2}$. Each point evaluation is easily verified to be a bounded linear functional on $b^{2}$. Hence, for each $z \in \mathbb{D}$, there exists a unique reproducing kernel $R_{z} \in b^{2}$ such that for every $\varphi \in L^{2}, Q \varphi(z)=\left\langle\varphi, R_{z}\right\rangle, z \in \mathbb{D}$, where $Q$ is the orthogonal projection from $L^{2}$ onto $b^{2}$.

As is well known, a function $u$ in $\mathbb{D}$ is harmonic if and only if it admits a decomposition $u=f+\bar{g}$ where $f, g$ are holomorphic on $\mathbb{D}$. Furthermore, if $u$ is in $L^{2}$, then both $f$ and $g$ are in $L_{a}^{2}$. Here, $L_{a}^{2}$ denotes the well known

Received June 7, 2014; received in final form January 29, 2016.

The first author was supported by NSFC (Nos. 11201274, 11471113) and ZJNSFC (No. LY14A010013).

The second author was supported by NSFC (No. 11101079).

2010 Mathematics Subject Classification. Primary 47B35. Secondary 31A05. 
holomorphic Bergman space consisting of all holomorphic functions in $L^{2}$. As a result of this observation, we see that

$$
b^{2}=z L_{a}^{2} \oplus \overline{L_{a}^{2}} .
$$

In particular, there is a simple relation between $R_{z}$ and the well known Bergman reproducing kernel $K_{z}$,

$$
R_{z}=K_{z}+\overline{K_{z}}-1, \quad z \in \mathbb{D},
$$

where $K_{z}(w)=\frac{1}{(1-w \bar{z})^{2}}$. Thus, $Q: L^{2} \rightarrow b^{2}$ admits the integral representation

$$
Q \varphi(z)=\int_{\mathbb{D}}\left[\frac{1}{(1-w \bar{z})^{2}}+\frac{1}{(1-z \bar{w})^{2}}-1\right] \varphi(w) d A(w)
$$

for $\varphi \in L^{2}$. Recall that the Bergman projection $P$, the orthogonal projection from $L^{2}$ onto $L_{a}^{2}$, has the following integral formula

$$
P \varphi(z)=\int_{\mathbb{D}} \frac{\varphi(w)}{(1-z \bar{w})^{2}} d A(w), \quad z \in \mathbb{D}
$$

for $\varphi \in L^{2}$. Hence, the projection $Q$ can be rewritten as

$$
Q \varphi=P(\varphi)+\overline{P(\bar{\varphi})}-P(\varphi)(0), \quad \varphi \in L^{2} .
$$

Let $U: L^{2} \rightarrow L^{2}$ be the operator $U f(z)=f(\bar{z})$, then $U$ is unitary and $U Q=Q U$. Given a function $\phi \in L^{2}$, the Toeplitz operator $T_{\phi}: b^{2} \rightarrow b^{2}$ and small Hankel operator $H_{\phi}: b^{2} \rightarrow b^{2}$ with symbol $\phi$ are densely defined by

$$
T_{\phi} u=Q(\phi u), \quad H_{\phi} u=Q U(\phi u)
$$

for functions $u \in b^{2}$. Clearly, $T_{\phi}$ and $H_{\phi}$ are both bounded linear operator on $b^{2}$ when $\phi \in L^{\infty}$. From the equality $U Q=Q U$, one can easily get the following relation between Toeplitz operator and small Hankel operator

$$
H_{\phi}=U T_{\phi}=T_{U \phi} U .
$$

For $\phi \in L^{2}$, the Toeplitz operator $\widetilde{T}_{\phi}$ and small Hankel operator $\widetilde{H}_{\phi}$ on the Bergman space $L_{a}^{2}$ are densely defined by

$$
\widetilde{T}_{\phi}(F)=P(\varphi F), \quad \widetilde{H}_{\phi} F=P U(\phi F), \quad F \in L_{a}^{2} .
$$

It is easy to see $P T_{\phi} P=\widetilde{T}_{\phi}$ and $P H_{\phi} P=\widetilde{H}_{\phi}$. Note $\widetilde{T}_{\phi}=0$ only if $\phi=0$. Combining these facts with formula (2), we have

$$
H_{\phi}=0 \Longleftrightarrow T_{\phi}=0 \Longleftrightarrow \phi=0 .
$$

Let $\phi^{*}(z)=\overline{\phi(\bar{z})}$, then $\phi^{*}=\overline{U \phi}=U \bar{\phi}$. It is easy to check $H_{\phi}^{*}=H_{\phi^{*}}$ and $\widetilde{H}_{\phi}^{*}=\widetilde{H}_{\phi^{*}}$. So $H_{\phi}$ is self-adjoint if and only if $\phi^{*}=\phi$.

The algebraic properties, such as commutativity or product of two operators, are important topics in operator theory. In [3], Brown and Halmos completely characterized the algebraic properties of Toeplitz operators on the 
classical Hardy space $H^{2}$. From then on, many related works on Toeplitz operators or (small) Hankel operators emerged (see [21], [13], for example).

For the case on the Bergman space of the unit disk, the algebraic properties are more subtle than that on the Hardy space. [1] and [2] obtained the BrownHalmos type theorems for Toeplitz operators with harmonic symbols. Many subsequent works studied these problems for special symbol classes, such as harmonic symbols, radial symbols or quasihomogeneous symbols, see [6], [17], [19], [20] for example. For boundedness, compactness, commutativity and product of small Hankel operators on the Bergman space, one is referred to [15], [23].

On the harmonic Bergman space, [14] has studied the algebra of Toeplitz operators and small Hankel operators. Some studies focusing on the algebraic properties of Toepltz operators with harmonic symbols [5], [7] or quasihomogeneous symbols [8], [9], [10], [18] showed that the results obtained are also quite different from the case on the Hardy or Bergman space.

Motivated by these works, in this paper we first study the algebraic properties of two small Hankel operators on the harmonic Bergman space. Unlike the Toeplitz operators case in which many algebraic properties are still unknown even for harmonic symbols or quasihomogeneous symbols, we obtain complete characterizations of commuting problem and the product problem for certain quasihomogeneous or harmonic symbols. Moreover, our results can apply to answer some algebraic properties of Toeplitz operators on the harmonic Bergman space.

In order to introduce our main results, we need the notation for quasihomogeneous functions. Let $\mathfrak{R}$ be the space of radial functions in $L^{2}$. By using the fact that the trigonometric polynomials are dense in $L^{2}$ and that for $k_{1} \neq k_{2}$, $e^{i k_{1} \theta} \mathfrak{R}$ is orthogonal to $e^{i k_{2} \theta} \mathfrak{R}$, one sees that

$$
L^{2}=\bigoplus_{k \in \mathbb{Z}} e^{i k \theta} \Re
$$

Thus, each function $f \in L^{2}$ can be written as (see [4]):

$$
\psi\left(r e^{i \theta}\right)=\sum_{k \in \mathbb{Z}} e^{i k \theta} \psi_{k}(r), \quad \psi_{k} \in \mathfrak{R} .
$$

Moreover, if $\psi \in L^{\infty}$, then each $\psi_{k}$ is also bounded on $\mathbb{D}$ since for each $r \in$ $[0,1)$,

$$
\left|\psi_{k}(r)\right|=\left|\frac{1}{2 \pi} \int_{0}^{2 \pi} \psi\left(r e^{i \theta}\right) e^{-i k \theta} d \theta\right| \leq \sup _{z \in \mathbb{D}}|\psi(z)|, \quad k \in \mathbb{Z} .
$$

We call every function in $e^{i k \theta} \mathfrak{R}$ to be quasihomonegeous function of degree $k$.

For the commutativity of two small Hankel operators, we first note that there is an obvious case. In fact, by (2) and (3), we have

$$
H_{1} H_{\phi}=H_{\phi} H_{1} \Longleftrightarrow T_{\phi}=T_{U \phi} \Longleftrightarrow \phi=U \phi
$$


then $H_{1} H_{\phi}=H_{U \phi} H_{1}$, and hence

$$
H_{1} H_{\phi+U \phi}=H_{\phi+U \phi} H_{1} .
$$

The above case will be included in our characterization of commuting problem for two small Hankel operators.

Now we state our main results of this paper.

TheOREm 1. Let $p \in \mathbb{Z}, \varphi \in L^{\infty}$ be radial and $\psi \in L^{\infty}$. Then $H_{e^{i p \theta} \varphi} H_{\psi}=$ $H_{\psi} H_{e^{i p \theta} \varphi}$ if and only if one of the followings holds:

(a) $\varphi=0$;

(b) $\psi$ is a quasihomonegeous function of degree $p$;

(c) $p=0, \varphi$ is nonzero constant and $U \psi=\psi$.

Note that $U \psi=\psi$ if and only if $\psi=\phi+U \phi$ with $\phi=\sum_{k=0}^{\infty} e^{i k \theta} \phi_{k}(r)$, where each $\phi_{k}$ is a radial function. In particular, if $\psi$ is harmonic, then $U \psi=\psi$ if and only if $\psi=\phi+U \phi$, where $\phi$ is an analytic function.

TheOREm 2. Let $u, v \in L^{\infty} \cap b^{2}$, then $H_{u} H_{v}=H_{v} H_{u}$ if and only if one of the followings holds:

(a) $\alpha u+\beta v=0$ for some $\alpha, \beta \in \mathbb{C}$ and $|\alpha|+|\beta| \neq 0$;

(b) $u=\alpha(\varphi+U \varphi)+\lambda_{1}, v=\beta(\varphi+U \varphi)+\lambda_{2}$, where $\alpha, \beta, \lambda_{1}, \lambda_{2} \in \mathbb{C}$ and $\varphi$ is an analytic function.

We would like to point out that the above main results can be applied to answer when the difference of product of Toeplitz operators $T_{\varphi} T_{\psi}-T_{U \psi} T_{U \varphi}$ is zero for one symbol being bounded and another being quasihomonegeous, or both being harmonic, since by $(2)$, we have

$$
U\left(T_{\varphi} T_{\psi}-T_{U \psi} T_{U \varphi}\right) U=H_{\varphi} H_{U \psi}-H_{U \psi} H_{\varphi}
$$

Theorem 3. Let $p \in \mathbb{Z}, \varphi \in L^{\infty}$ be radial and $\psi, \phi \in L^{\infty}$. Then the following statements are equivalent:

(a) $H_{e^{i p \theta} \varphi} H_{\psi}=H_{\phi}$

(b) $H_{\psi} H_{e^{i p \theta} \varphi}=H_{\phi}$;

(c) $\varphi=\phi=0$ or $\psi=\phi=0$.

THEOREM 4. Let $u, v, \phi \in L^{\infty} \cap b^{2}$. Then the following statements are equivalent:

(a) $H_{u} H_{v}=H_{\phi}$;

(b) $H_{v} H_{u}=H_{\phi}$;

(c) $u=\phi=0$ or $v=\phi=0$.

Also, by (2) the above two results can be applied to answer when $H_{\varphi} T_{\psi}=$ $T_{\phi}$ or $T_{\psi} H_{\varphi}=T_{\phi}$ for $\varphi, \psi$ and $\phi$ in certain symbol classes.

In the next two sections, we will prove Theorems 1 and 2 respectively, and in the last section, we will prove Theorems 3 and 4. Many corollaries including semi-commuting problem and zero product problem will also be included in corresponding sections. 


\section{Commutativity of two small Hankel operators with quasihomonegeous symbols}

In this section, we are going to prove Theorem 1 . We first give the following preparations.

For $\varphi \in L^{1}([0,1), r d r)$, the Mellin transform $\widehat{\varphi}$ is defined by the equation

$$
\widehat{\varphi}(z)=\int_{0}^{1} \varphi(r) r^{z-1} d r
$$

It is clear that $\widehat{\varphi}$ is analytic in the right half plane $\{z: \operatorname{Re} z>2\}$. It is important and helpful to know that the Mellin transform is uniquely determined by its value on an arithmetic sequence of integers. In fact, we have the following classical theorem (see [22], p. 102).

LEMma 5. Suppose that $\varphi$ is a bounded analytic function on $\{z: \operatorname{Re} z>0\}$. If $\varphi$ vanishes at the pairwise distinct points $\left\{z_{k}: k=1,2, \ldots\right\}$, where $\inf \left\{\left|z_{k}\right|\right\}>0$ and $\sum_{k=1}^{\infty} \operatorname{Re}\left(\frac{1}{z_{k}}\right)=\infty$, then $\varphi=0$.

REMARK 6. As a simple application of the above lemma, we have the following fact which we will use frequently to prove our main results: for $\varphi \in$ $L^{1}([0,1), r d r)$, if there exists a sequence $\left\{n_{k}\right\}_{k \geq 0} \subset \mathbb{N}$ such that $\sum_{k \geq 0} \frac{1}{n_{k}}=\infty$ and $\widehat{\varphi}\left(n_{k}\right)=0$, then $\varphi=0$.

When considering the product of two small Hankel operators, we also use a well known fact about the Mellin convolution of their symbols. If $\varphi, \psi \in$ $L^{1}([0,1), r d r)$, their Mellin convolution is defined by

$$
\varphi * \psi(r)=\int_{r}^{1} \varphi\left(\frac{r}{t}\right) \psi(t) \frac{d t}{t}, \quad r \in[0,1) .
$$

It is easy to verify that

$$
\widehat{\varphi * \psi}(s)=\widehat{\varphi}(s) \widehat{\psi}(s)
$$

and that if $\varphi$ and $\psi$ are both in $L^{1}([0,1), r d r)$, so is $\varphi * \psi$.

The following lemma comes from [10, Lemma 2.1].

LEMMA 7. Let $p \in \mathbb{Z}$ and $\varphi$ be a bounded radial function. Then for each $k \in \mathbb{N}$,

$$
\begin{aligned}
& T_{e^{i p \theta} \varphi}\left(z^{k}\right)= \begin{cases}2(k+p+1) \hat{\varphi}(2 k+p+2) z^{k+p}, & k+p \geq 0, \\
2(-k-p+1) \hat{\varphi}(-p+2) \bar{z}^{-k-p}, & k+p<0,\end{cases} \\
& T_{e^{i p \theta} \varphi}\left(\bar{z}^{k}\right)= \begin{cases}2(k-p+1) \hat{\varphi}(2 k-p+2) \bar{z}^{k-p}, & k-p>0, \\
2(p-k+1) \hat{\varphi}(p+2) z^{p-k}, & k-p \leq 0 .\end{cases}
\end{aligned}
$$

By (2) and the above lemma, we have the following result which will be used frequently in this and the last sections. 
LEMMA 8. Let $p \in \mathbb{Z}$ and $\varphi$ be a bounded radial function. Then for each $k \in \mathbb{N}$,

$$
\begin{aligned}
& H_{e^{i p \theta} \varphi}\left(z^{k}\right)= \begin{cases}2(k+p+1) \hat{\varphi}(2 k+p+2) \bar{z}^{k+p}, & k+p>0, \\
2(-k-p+1) \hat{\varphi}(-p+2) z^{-k-p}, & k+p \leq 0,\end{cases} \\
& H_{e^{i p \theta} \varphi}\left(\bar{z}^{k}\right)= \begin{cases}2(k-p+1) \hat{\varphi}(2 k-p+2) z^{k-p}, & k-p \geq 0, \\
2(p-k+1) \hat{\varphi}(p+2) \bar{z}^{p-k}, & k-p<0 .\end{cases}
\end{aligned}
$$

REMARK 9. Let $p, k \in \mathbb{Z}$ and $\varphi$ be radial. In view of the above two lemmas, we see that there are two constants $\lambda, \mu$, independent on $z=r e^{i \theta}$, such that

$$
T_{e^{i p \theta} \varphi}\left(r^{|k|} e^{i k \theta}\right)=\lambda r^{|p+k|} e^{i(p+k) \theta}, \quad H_{e^{i p \theta} \varphi}\left(r^{|k|} e^{i k \theta}\right)=\mu r^{|p+k|} e^{-i(p+k) \theta} .
$$

Now we first investigate commutativity of two small Hankel operators with quasihomogeneous symbols.

Proposition 10. Let $p, q \in \mathbb{Z}$ and $\varphi, \psi$ be bounded and radial. Then $H_{e^{i p \theta} \varphi}$ commutes with $H_{e^{i q \theta} \psi}$ if and only if one of the following cases holds:

(a) $p=q$;

(b) $p \neq q$ but $\varphi=0$ or $\psi=0$.

Proof. Suppose $p \neq q$. By Lemma 8, direct calculation gives that for each integer $k \geq|p|+|q|, H_{e^{i p \theta} \varphi} H_{e^{i q \theta} \psi}\left(z^{k}\right)$ and $H_{e^{i q \theta} \psi} H_{e^{i p \theta} \varphi}\left(z^{k}\right)$ equal to

$$
4(k+q+1)(k+q-p+1) \hat{\psi}(2 k+q+2) \hat{\varphi}(2 k+2 q-p+2) z^{k+q-p}
$$

and

$$
4(k+p+1)(k+p-q+1) \hat{\varphi}(2 k+p+2) \hat{\psi}(2 k+2 p-q+2) z^{k+p-q}
$$

respectively. Since $p \neq q$, we see $k+p-q \neq k+q-p$. Hence, if $H_{e^{i p \theta} \varphi}$ commutes with $H_{e^{i q \theta} \psi}$, from the above two equations we have

$$
\hat{\varphi}(2 k+p+2) \hat{\psi}(2 k+2 p-q+2)=0, \quad k \geq|p|+|q| .
$$

Let $E_{1}=\{k>0: \hat{\varphi}(2 k+p+2)=0\}, E_{2}=\{k>0: \hat{\psi}(2 k+2 p-q+2)=0\}$. Then it is easy to see that $\sum_{k \in E_{1}} \frac{1}{k}=\infty$ or $\sum_{k \in E_{2}} \frac{1}{k}=\infty$, hence by Remark 6 we get $\varphi=0$ or $\psi=0$.

For the case $p=q$, direct computation using Lemma 8 gives that for each integer $k \geq 0$,

$$
\begin{aligned}
& H_{e^{i p \theta} \varphi} H_{e^{i p \theta} \psi}\left(z^{k}\right)=H_{e^{i p \theta} \psi} H_{e^{i p \theta} \varphi}\left(z^{k}\right), \\
& H_{e^{i p \theta} \varphi} H_{e^{i p \theta} \psi}\left(\bar{z}^{k}\right)=H_{e^{i p \theta} \psi} H_{e^{i p \theta} \varphi}\left(\bar{z}^{k}\right),
\end{aligned}
$$

hence $H_{e^{i p \theta} \varphi}$ commutes with $H_{e^{i q \theta} \psi}$. We complete the proof.

The above result implies that two small Hankel operators with quasihomogeneous symbols of same degrees always commute, while those with quasihomogeneous of different degrees commute only in the trivial case. In particular, 
$H_{e^{i p \theta} \varphi}$ is a normal operator. $H_{e^{i p \theta} \varphi}$ is self-adjoint if and only if $\varphi$ is a realvalued radial function for $H_{e^{i p \theta} \varphi}^{*}=H_{e^{i p \theta} \bar{\varphi}}$. These facts are similar to the commuting small Hankel operators with quasihomogeneous symbols on the Bergman space [15], [20], but different from the commuting Toeplitz operators on the Bergman space [19] or on the harmonic Bergman space [8] with quasihomogeneous symbols.

Proof of Theorem 1. The sufficiency is obvious by (6) and Proposition 10. We turn to show the necessity. Let $\psi(z)=\sum_{k \in \mathbb{Z}} e^{i k \theta} \psi_{k}(r)$.

Claim: $H_{e^{i p \theta} \varphi} H_{\psi}$ commutes with $H_{\psi} H_{e^{i p \theta} \varphi}$ if and only if

$$
H_{e^{i p \theta} \varphi} H_{e^{i(p+n) \theta} \psi_{p+n}}=H_{e^{i(p-n) \theta} \psi_{p-n}} H_{e^{i p \theta} \varphi}, \quad n \in \mathbb{Z} .
$$

Indeed, for fixed $k_{1}, k_{2} \in \mathbb{Z}$, by Remark 9 we have

$$
\begin{aligned}
& \left\langle H_{e^{i p \theta} \varphi} H_{e^{i k \theta} \psi_{k}}\left(r^{\left|k_{1}\right|} e^{i k_{1} \theta}\right), r^{\left|k_{2}\right|} e^{i k_{2} \theta}\right\rangle \\
& \quad= \begin{cases}0, & k \neq p+k_{2}-k_{1}, \\
\left\langle H_{e^{i p \theta} \varphi} H_{e^{i\left(p+k_{2}-k_{1}\right) \theta} \psi_{p+k_{2}-k_{1}}}\left(r^{\left|k_{1}\right|} e^{i k_{1} \theta}\right), r^{\left|k_{2}\right|} e^{i k_{2} \theta}\right\rangle, & k=p+k_{2}-k_{1},\end{cases}
\end{aligned}
$$

and

$$
\begin{aligned}
& \left\langle H_{e^{i k \theta} \psi_{k}} H_{e^{i p \theta} \varphi}\left(r^{\left|k_{1}\right|} e^{i k_{1} \theta}\right), r^{\left|k_{2}\right|} e^{i k_{2} \theta}\right\rangle \\
& \quad= \begin{cases}0, & k \neq p+k_{1}-k_{2}, \\
\left\langle H_{e^{i\left(p+k_{1}-k_{2}\right) \theta} \psi_{p+k_{1}-k_{2}}} H_{e^{i p \theta} \varphi}\left(r^{\left|k_{1}\right|} e^{i k_{1} \theta}\right), r^{\left|k_{2}\right|} e^{i k_{2} \theta}\right\rangle, & k=p+k_{1}-k_{2} .\end{cases}
\end{aligned}
$$

Hence for $k_{1}, k_{2} \in \mathbb{Z}$, we have

$$
\begin{aligned}
& \left\langle H_{e^{i p \theta} \varphi} H_{\psi}\left(r^{\left|k_{1}\right|} e^{i k_{1} \theta}\right), r^{\left|k_{2}\right|} e^{i k_{2} \theta}\right\rangle \\
& \quad=\left\langle H_{e^{i p \theta} \varphi} H_{e^{i\left(p+k_{2}-k_{1}\right) \theta} \psi_{p+k_{2}-k_{1}}}\left(r^{\left|k_{1}\right|} e^{i k_{1} \theta}\right), r^{\left|k_{2}\right|} e^{i k_{2} \theta}\right\rangle
\end{aligned}
$$

and

$$
\begin{aligned}
& \left\langle H_{\psi} H_{e^{i p \theta} \varphi}\left(r^{\left|k_{1}\right|} e^{i k_{1} \theta}\right), r^{\left|k_{2}\right|} e^{i k_{2} \theta}\right\rangle \\
& \quad=\left\langle H_{e^{i\left(p+k_{1}-k_{2}\right) \theta} \psi_{p+k_{1}-k_{2}}} H_{e^{i p \theta} \varphi}\left(r^{\left|k_{1}\right|} e^{i k_{1} \theta}\right), r^{\left|k_{2}\right|} e^{i k_{2} \theta}\right\rangle .
\end{aligned}
$$

Let $n=k_{2}-k_{1}$, then $n \in \mathbb{Z}$. So $H_{e^{i p \theta} \varphi} H_{\psi}=H_{\psi} H_{e^{i p \theta} \varphi}$ together with the above two equations give

$$
\begin{aligned}
& \left\langle H_{e^{i p \theta} \varphi} H_{e^{i(p+n) \theta} \psi_{p+n}}\left(r^{\left|k_{1}\right|} e^{i k_{1} \theta}\right), r^{\left|k_{1}+n\right|} e^{i\left(k_{1}+n\right) \theta}\right\rangle \\
& \quad=\left\langle H_{e^{i(p-n) \theta} \psi_{p-n}} H_{e^{i p \theta} \varphi}\left(r^{\left|k_{1}\right|} e^{i k_{1} \theta}\right), r^{\left|k_{1}+n\right|} e^{i\left(k_{1}+n\right) \theta}\right\rangle
\end{aligned}
$$

for all $n, k_{1} \in \mathbb{Z}$. Now fix $n \in \mathbb{Z}$. It follows from Remark 9 again that for each $m \in \mathbb{Z}$ and $m \neq n$,

$$
\left\langle H_{e^{i p \theta} \varphi} H_{e^{i(p+n) \theta} \psi_{p+n}}\left(r^{\left|k_{1}\right|} e^{i k_{1} \theta}\right), r^{\left|k_{1}+m\right|} e^{i\left(k_{1}+m\right) \theta}\right\rangle=0
$$

and

$$
\left\langle H_{e^{i(p-n) \theta} \psi_{p-n}} H_{e^{i p \theta} \varphi}\left(r^{\left|k_{1}\right|} e^{i k_{1} \theta}\right), r^{\left|k_{1}+m\right|} e^{i\left(k_{1}+m\right) \theta}\right\rangle=0
$$


for all $k_{1} \in \mathbb{Z}$. Therefore, it is easy to see that (8) holds by the above two equations together with (9). The converse is obvious from the above arguments, so the claim holds.

In order to prove the necessity, in what follows we assume $\varphi \neq 0$ and $\psi_{m_{0}} \neq$ 0 for some $m_{0} \neq 0$ and $m_{0} \neq p$. We want to use the equation (8) to deduce $p=0, \varphi$ is a nonzero constant and $\psi_{m_{0}}=\psi_{-m_{0}}$, which is the case (c) of the theorem and will finish the proof.

Let $n_{0}=p-m_{0}$, then $n_{0} \neq 0, p$ and $\psi_{p-n_{0}} \neq 0$. By (8) we have

$$
H_{e^{i p \theta} \varphi} H_{e^{i\left(p+n_{0}\right) \theta} \psi_{p+n_{0}}}\left(z^{k}\right)=H_{e^{i\left(p-n_{0}\right) \theta} \psi_{p-n_{0}}} H_{e^{i p \theta} \varphi}\left(z^{k}\right)
$$

and

$$
H_{e^{i p \theta} \varphi} H_{e^{i\left(p+n_{0}\right) \theta} \psi_{p+n_{0}}}\left(\bar{z}^{k}\right)=H_{e^{i\left(p-n_{0}\right) \theta} \psi_{p-n_{0}}} H_{e^{i p \theta} \varphi}\left(\bar{z}^{k}\right) .
$$

By Lemma 8 and straightforward calculations, the above two equations give that for all $k \geq|p|+\left|n_{0}\right|$,

$$
\begin{aligned}
& \left(k+p+n_{0}+1\right) \widehat{\varphi}\left(2 k+p+2 n_{0}+2\right) \widehat{\psi_{p+n_{0}}}\left(2 k+p+n_{0}+2\right) \\
& \quad=(k+p+1) \widehat{\varphi}(2 k+p+2) \widehat{\psi_{p-n_{0}}}\left(2 k+p+n_{0}+2\right)
\end{aligned}
$$

and

$$
\begin{aligned}
\left(k-p-n_{0}+1\right) \widehat{\varphi}\left(2 k-p-2 n_{0}+2\right) \widehat{\psi_{p+n_{0}}}\left(2 k-p-n_{0}+2\right) \\
\quad=(k-p+1) \widehat{\varphi}(2 k-p+2) \widehat{\psi_{p-n_{0}}}\left(2 k-p-n_{0}+2\right),
\end{aligned}
$$

respectively. Substituting $k$ with $k+p+n_{0}$ in the last equation above, we get

$$
\begin{aligned}
& (k+1) \widehat{\varphi}(2 k+p+2) \widehat{\psi_{p+n_{0}}}\left(2 k+p+n_{0}+2\right) \\
& \quad=\left(k+n_{0}+1\right) \widehat{\varphi}\left(2 k+p+2 n_{0}+2\right) \widehat{\psi_{p-n_{0}}}\left(2 k+p+n_{0}+2\right)
\end{aligned}
$$

for all $k \geq 2|p|+2\left|n_{0}\right|$. Let

$$
\begin{aligned}
& E_{1}=\{k>0: \widehat{\varphi}(2 k+p+2)=0\}, \\
& E_{2}=\left\{k>0: \widehat{\psi_{p-n_{0}}}\left(2 k+p+n_{0}+2\right)=0\right\} .
\end{aligned}
$$

Note $\varphi \neq 0$ and $\psi_{p-n_{0}} \neq 0$. Then by Remark 6 , we see $\sum_{k \in E_{1}} \frac{1}{k}<\infty$ and $\sum_{k \in E_{2}} \frac{1}{k}<\infty$. Put

$$
E=\left(\mathbb{Z}-E_{1} \cup E_{2}\right) \cap\left[2|p|+2\left|n_{0}\right|,+\infty\right) .
$$

Then $\sum_{k \in E} \frac{1}{k}=\infty$. Since for $k \in E, \widehat{\varphi}(2 k+p+2) \neq 0, \widehat{\psi}\left(2 k+p+2 n_{0}+2\right) \neq 0$, then (10) or (11) tells $\widehat{\psi_{p+n_{0}}}\left(2 k+p+n_{0}+2\right) \neq 0$ when $k \in E$. Thus by (10) and (11), we get

$$
\begin{aligned}
& \left(k+n_{0}+1\right)\left(k+p+n_{0}+1\right)\left[\widehat{\varphi}\left(2 k+p+2 n_{0}+2\right)\right]^{2} \\
& \quad=(k+1)(k+p+1)[\widehat{\varphi}(2 k+p+2)]^{2}
\end{aligned}
$$


for all $k \in E$. Let $z=2 k+2$, then the above equality becomes

$$
\left(z+2 n_{0}\right)\left(z+2 p+2 n_{0}\right)\left[\widehat{\varphi}\left(z+p+2 n_{0}\right)\right]^{2}=z(z+2 p)[\widehat{\varphi}(z+p)]^{2} .
$$

It follows from Lemma 5 and (12) that the above holds on $\{z: \operatorname{Re} z \geq 2|p|+$ $\left.2\left|n_{0}\right|\right\}$. Without loss of generality, we now assume $n_{0}>0$. So put $z=2 k n_{0}$ above with $k \geq 2|p|+2\left|n_{0}\right|$, then

$$
2 k n_{0}\left(2 k n_{0}+2 p\right)\left[\widehat{\varphi}\left(2 k n_{0}+p\right)\right]^{2}=2 k_{0} n_{0}\left(2 k_{0} n_{0}+2 p\right)\left[\widehat{\varphi}\left(2 k_{0} n_{0}+p\right)\right]^{2}
$$

for all $k \geq k_{0}=2|p|+2\left|n_{0}\right|$. Let $C=2 k_{0} n_{0}\left(2 k_{0} n_{0}+2 p\right)\left[\widehat{\varphi}\left(2 k_{0} n_{0}+p\right)\right]^{2}$, then clearly $C \neq 0$. Now using the property of Mellin convolution (7), the above equation becomes

$$
\widehat{\varphi * \varphi}\left(2 k n_{0}+p\right)=\frac{C}{2 k n_{0}\left(2 k n_{0}+2 p\right)}=C r^{p * r^{-p}}\left(2 k n_{0}+p\right)
$$

for $k \geq k_{0}$. Therefore by Remark 6 , we get

$$
\varphi * \varphi=C r^{p} * r^{-p} .
$$

Since $\varphi$ is bounded, so it is easy to check $\varphi * \varphi \in L^{2}([0,1), r d r)$, which forces $r^{p} * r^{-p}$ to be in $L^{2}([0,1), r d r)$. But it holds only when $p=0$ since $r^{p} * r^{-p}(s)=\frac{1}{2 p}\left(s^{-p}-s^{p}\right)$ for $p \neq 0$. Hence, we get $p=0$. So $\varphi * \varphi=C 1 * 1$ which gives $\widehat{\varphi}^{2}=C \widehat{1}^{2}$ by (7), that is, $\widehat{\varphi}= \pm \sqrt{C} \widehat{1}$. By Remark 6 , we easily get that $\varphi=\sqrt{C}$ or $\varphi=-\sqrt{C}$, a nonzero constant.

Note $n_{0}=p-m_{0}$ and $\widehat{1}(k)=1 / k$. Now when $p=0$ and $\varphi$ is a nonzero constant, (10) or (11) will give

$$
\widehat{\psi_{m_{0}}}\left(2 k+m_{0}+2\right)=\widehat{\psi_{-m_{0}}}\left(2 k+m_{0}+2\right), \quad k \in E .
$$

So by Remark 6 again, we obtain that $\psi_{m_{0}}=\psi_{-m_{0}}$. The proof is complete.

\section{Commutativity of two small Hankel operators with harmonic symbols}

In this section, we are going to prove Theorem 2. We always assume $u=f+\bar{g}, v=h+\bar{k}$, where $f, g, h, k$ are all holomorphic functions on $\mathbb{D}$ with $g(0)=k(0)=0$.

Recall $U \varphi(z)=\varphi(\bar{z})$ and $\varphi^{*}=\overline{U \varphi}=U \bar{\varphi}$, so it is easy to check that $\left(K_{z}\right)^{*}=$ $K_{\bar{z}}$ or $\overline{K_{z}}=U K_{\bar{z}}$ and for $\varphi \in L^{2},[P(\varphi)]^{*}=P\left(\varphi^{*}\right)$.

Note $b^{2}=z L_{a}^{2} \oplus \overline{L_{a}^{2}}$. Let $\widetilde{U}$ be the unitary operator from $z L_{a}^{2} \oplus \overline{L_{a}^{2}}$ to $z L_{a}^{2} \oplus L_{a}^{2}$ defined by

$$
\widetilde{U}=\left(\begin{array}{cc}
I & 0 \\
0 & U
\end{array}\right)
$$


It is easy to see $\widetilde{U}^{*}$ maps $z L_{a}^{2} \oplus L_{a}^{2}$ to $L_{h}^{2}=z L_{a}^{2} \oplus \overline{L_{a}^{2}}$ and

$$
\widetilde{U}^{*}=\left(\begin{array}{cc}
I & 0 \\
0 & U
\end{array}\right)
$$

since $U^{*}=U$ on $L^{2}$. For $\varphi$ and $\psi$ in $L^{2}$, we let $\varphi \otimes \psi$ be a operator defined by $\varphi \otimes \psi(\phi)=\langle\phi, \psi\rangle \varphi$ for function $\phi \in L^{2}$.

We use the idea of [14] to obtain the following block matrix representation of small Hankel operator. The representation is useful in this paper and indicates that the small Hankel operators on the harmonic Bergman space are closely related to the Toeplitz operators and small Hankel operators on the Bergman space.

Lemma 11. Let $\phi \in L^{\infty}$. Then on $z L_{a}^{2} \oplus L_{a}^{2}$, we have

$$
\widetilde{U} H_{\phi} \widetilde{U}^{*}=\left(\begin{array}{cc}
\widetilde{H}_{\phi}-1 \otimes P(\bar{\phi}) & \widetilde{T}_{U \phi}-\underset{\widetilde{H}_{U \phi}}{1 \otimes P\left(\phi^{*}\right)} \\
\widetilde{T}_{\phi} & \widetilde{H}_{U}
\end{array}\right) .
$$

Proof. Since $\overline{P(\bar{\varphi})}=U[P(\bar{\varphi})]^{*}=U P\left(\bar{\varphi}^{*}\right)=U P U \varphi$ for $\varphi \in L^{2}$, so by (1), it is easy to see that $Q=P-1 \otimes 1+U P U$. Now for $f_{1} \in z L_{a}^{2}$ and $f_{2} \in L_{a}^{2}$, we see that

$$
H_{\phi} f_{1}=Q\left(U \phi U f_{1}\right)=\widetilde{H}_{\phi} f_{1}-[1 \otimes P(\bar{\phi})] f_{1}+U \widetilde{T}_{\phi} f_{1}
$$

and

$$
H_{\phi} U f_{2}=Q\left(U \phi f_{2}\right)=\widetilde{T}_{U \phi} f_{2}-\left[1 \otimes P\left(\phi^{*}\right)\right] f_{2}+U \widetilde{H}_{U \phi} f_{2},
$$

so for $\left[f_{1}, f_{2}\right]^{T}$ in $z L_{a}^{2} \oplus L_{a}^{2}$, the above calculation shows

$$
\begin{aligned}
\widetilde{U} H_{\phi} \widetilde{U}^{*}\left(\begin{array}{l}
f_{1} \\
f_{2}
\end{array}\right) & =\widetilde{U} H_{\phi}\left(\begin{array}{c}
f_{1} \\
U f_{2}
\end{array}\right) \\
& =\widetilde{U}\left(\begin{array}{c}
{\left[\widetilde{H}_{\phi}-1 \otimes P(\bar{\phi})\right] f_{1}+\left[\widetilde{T}_{U \phi}-1 \otimes P\left(\phi^{*}\right)\right] f_{2}} \\
U \widetilde{T}_{\phi} f_{1}+U \widetilde{H}_{U \phi} f_{2}
\end{array}\right) \\
& =\left(\begin{array}{c}
{\left[\widetilde{H}_{\phi}-1 \otimes P(\bar{\phi})\right] f_{1}+\left[\widetilde{T}_{U \phi}-1 \otimes P\left(\phi^{*}\right)\right] f_{2}} \\
\widetilde{T}_{\phi} f_{1}+\widetilde{H}_{U \phi} f_{2}
\end{array}\right),
\end{aligned}
$$

which gives the desired matrix representation. The proof is complete.

We recall some simple facts about Toeplitz operator $\widetilde{T}_{\varphi}$ and small Hankel operator $\widetilde{H}_{\phi}$ on the Bergman space $L_{a}^{2}$. It is clear that $\widetilde{H}_{\phi}=\widetilde{H}_{U P U \phi}$ and $\widetilde{H}_{1}=1 \otimes 1$. In addition, for analytic functions $\varphi$, we have $\widetilde{T}_{\bar{\varphi}} K_{z}=\overline{\varphi(z)} K_{z}$ and

$$
\widetilde{T}_{U \varphi} \widetilde{H}_{\psi}=\widetilde{H}_{\psi} \widetilde{T}_{\varphi}=\widetilde{H}_{\varphi \psi} .
$$

The first equality above comes from [15, Proposition 2.1] and the second one is deduced by direct computation.

The following lemma gives key formula to prove Theorem 2. 
LEMMA 12. If $H_{u}$ commutes with $H_{v}$, then

$$
P\left(k U g-g U k+f^{*} \bar{h}-h^{*} \bar{f}\right)=g h^{*}-k f^{*},
$$

and for each $z \in \mathbb{D}$,

$$
\begin{aligned}
& f^{*} P\left(k \overline{K_{z}}\right)+k(z) P\left(f^{*} \overline{K_{z}}\right)-P\left[\left(k f^{*}\right) \overline{K_{z}}\right]-k(z) f^{*} \\
& \quad=h^{*} P\left(g \overline{K_{z}}\right)+g(z) P\left(h^{*} \overline{K_{z}}\right)-P\left[\left(g h^{*}\right) \overline{K_{z}}\right]-g(z) h^{*} .
\end{aligned}
$$

Proof. By Lemma 11, we can get

$$
\widetilde{U} H_{u} H_{v} \widetilde{U}^{*}=\widetilde{U} H_{u} \widetilde{U}^{*} \widetilde{U} H_{v} \widetilde{U}^{*}=\left(\begin{array}{cc}
A & B \\
C & D
\end{array}\right),
$$

where $C: z L_{a}^{2} \rightarrow L_{a}^{2}$ and

$$
C=\widetilde{T}_{u}\left[\widetilde{H}_{v}-1 \otimes P(\bar{v})\right]+\widetilde{H}_{U u} \widetilde{T}_{v} .
$$

So $\widetilde{U}\left(H_{u} H_{v}\right) \widetilde{U}^{*}=\widetilde{U}\left(H_{v} H_{u}\right) \widetilde{U}^{*}$ gives that

$$
\widetilde{T}_{u}\left[\widetilde{H}_{v}-1 \otimes P(\bar{v})\right]+\widetilde{H}_{U u} \widetilde{T}_{v}=\widetilde{T}_{v}\left[\widetilde{H}_{u}-1 \otimes P(\bar{u})\right]+\widetilde{H}_{U v} \widetilde{T}_{u} .
$$

We take adjoint of both sides of the above equality to get

$$
\left[\widetilde{H}_{v^{*}}-P(\bar{v}) \otimes 1\right] \widetilde{T}_{\bar{u}}+\widetilde{T}_{\bar{v}} \widetilde{H}_{\bar{u}}=\left[\widetilde{H}_{u^{*}}-P(\bar{u}) \otimes 1\right] \widetilde{T}_{\bar{v}}+\widetilde{T}_{\bar{u}} \widetilde{H}_{\bar{v}},
$$

then acting on $K_{\bar{z}}\left(=K_{z}^{*}\right)$ of the above and applying (13) and $g(0)=k(0)=0$, we can get

$$
\begin{aligned}
& f^{*} P\left(k \overline{K_{z}}\right)+k(z) P\left(f^{*} \overline{K_{z}}\right)+P\left[\left(f^{*} \bar{h}-h^{*} \bar{f} \overline{K_{z}}\right]-k(z) f^{*}\right. \\
& \quad=h^{*} P\left(g \overline{K_{z}}\right)+g(z) P\left(h^{*} \overline{K_{z}}\right)+P\left[(g U k-k U g) \overline{K_{z}}\right]-g(z) h^{*} .
\end{aligned}
$$

Put $z=0$ in the above equality, we get (14). Set $G=k U g-g U k+f^{*} \bar{h}-$ $h^{*} \bar{f}$. Since

$$
P\left[(I-P)(G) \overline{K_{z}}\right](w)=\left\langle(I-P)(G) \overline{K_{z}}, K_{w}\right\rangle=\left\langle(I-P)(G), K_{z} K_{w}\right\rangle=0,
$$

where $I$ is identity operator on $L^{2}$, hence by (14) we have

$$
P\left(G \overline{K_{z}}\right)=P\left[P(G) \overline{K_{z}}\right]+P\left[(I-P)(G) \overline{K_{z}}\right]=P\left[\left(g h^{*}-k f^{*}\right) \overline{K_{z}}\right] .
$$

Insert the above into (17) we then get (15), completing the proof.

It should be pointed out that, the information of commutativity of two small Hankel operators with harmonic symbols are all included in the equations (14) and (15), just as we will show below. It is easy to verify that for nonnegative integer $n$,

$$
P\left(u^{n} \overline{K_{z}}\right)(w)=\int_{\mathbb{D}} \frac{u^{n} d A(u)}{(1-z \bar{u})^{2}(1-w \bar{u})^{2}}=\frac{1}{n+1} \sum_{j=0}^{n} c_{n, j} z^{n-j} w^{j},
$$


where $c_{n, j}=(n+1-j)(j+1), 0 \leq j \leq n$. Now let $f(z)=\sum_{n=0}^{\infty} a_{n} z^{n}$, then applying the above formula we get

$$
P\left(f \overline{K_{z}}\right)(w)=\sum_{j=0}^{\infty} \sum_{s=0}^{\infty} \frac{a_{j+s}}{j+s+1} c_{j+s, j} z^{s} w^{j} .
$$

LEMMA 13. Let $u=f+\bar{g}, v=h+\bar{k}, f(z)=\sum_{n=0}^{\infty} a_{n} z^{n}, g(z)=\sum_{n=0}^{\infty} b_{n} z^{n}$, $h(z)=\sum_{n=0}^{\infty} c_{n} z^{n}$ and $k(z)=\sum_{n=0}^{\infty} d_{n} z^{n}$, where $b_{0}=d_{0}=0$. If $H_{u}$ commutes with $H_{v}$, then

$$
\sum_{k=1}^{n-1} \alpha_{j, k}\left(\overline{a_{k}} d_{n-k}-\overline{c_{k}} b_{n-k}\right)=0, \quad 1 \leq j \leq n-1,
$$

for each fixed $n \geq 2$, where

$$
\alpha_{j, k}= \begin{cases}\frac{(n-k)(j+1) j}{k+1}, & j \leq k, \\ \frac{(n-j)(n-j+1) k}{n+1-k}, & j>k .\end{cases}
$$

Proof. We deduce (19) from the equality (15). Indeed, direct calculations combining with the formula (18) give

$$
\begin{aligned}
f^{*}(w) P\left(k \overline{K_{z}}\right)(w) & =\sum_{t, s=0}^{\infty}\left(\sum_{j=0}^{t} \overline{a_{j}} d_{t+s-j} \frac{c_{t+s-j, t-j}}{t+s+1-j}\right) z^{s} w^{t}, \\
k(z) P\left(f^{*} \overline{K_{z}}\right)(w) & =\sum_{t, s=0}^{\infty}\left(\sum_{j=t}^{t+s} \overline{a_{j}} d_{t+s-j} \frac{c_{j, t}}{j+1}\right) z^{s} w^{t}
\end{aligned}
$$

and

$$
P\left(f^{*} k \overline{K_{z}}\right)(w)=\sum_{t, s=0}^{\infty}\left(\sum_{j=0}^{t+s} \overline{a_{j}} d_{t+s-j} \frac{c_{t+s, t}}{t+s+1}\right) z^{s} w^{t},
$$

hence in the expansion of the left-hand side of the equality (15), the coefficient of $w^{t}$ is

$$
\begin{gathered}
\sum_{s=0}^{\infty}\left[\sum_{j=0}^{t} \overline{a_{j}} d_{t+s-j} \frac{c_{t+s-j, t-j}}{t+s+1-j}\right] z^{s}+\sum_{s=0}^{\infty}\left[\sum_{j=t}^{t+s} \overline{a_{j}} d_{t+s-j} \frac{c_{j, t}}{j+1}\right] z^{s} \\
-\sum_{s=0}^{\infty}\left[\sum_{j=0}^{t+s} \overline{a_{j}} d_{t+s-j} \frac{c_{t+s, t}}{t+s+1}\right] z^{s}-\sum_{s=0}^{\infty} \overline{a_{t}} d_{s} z^{s}
\end{gathered}
$$

for each integer $t \geq 0$, that is,

$$
\begin{aligned}
\sum_{s=0}^{\infty} & {\left[\sum_{j=0}^{t} \overline{a_{j}} d_{t+s-j} \frac{c_{t+s-j, t-j}}{t+s+1-j}+\sum_{j=t}^{t+s} \overline{a_{j}} d_{t+s-j} \frac{c_{j, t}}{j+1}\right.} \\
& \left.-\sum_{j=0}^{t+s} \overline{a_{j}} d_{t+s-j} \frac{c_{t+s, t}}{t+s+1}-\overline{a_{t}} d_{s}\right] z^{s}
\end{aligned}
$$


for each $t \geq 0$. Recall that $c_{n, j}=(n+1-j)(j+1), 0 \leq j \leq n$, and $d_{0}=0$, then the above becomes

$$
\begin{aligned}
& \sum_{s=0}^{\infty} \frac{-1}{t+s+1}\left[\sum_{j=1}^{t} \overline{a_{j}} d_{t+s-j} \frac{j s(s+1)}{t+s+1-j}\right. \\
& \left.+\sum_{j=t+1}^{t+s-1} \overline{a_{j}} d_{t+s-j} \frac{t(t+1)(t+s-j)}{j+1}\right] z^{s}
\end{aligned}
$$

for each $t \geq 1$. Similar formula as above can be obtained when computing the right-hand side of (15), thus the equality (15) will give

$$
\begin{aligned}
& \sum_{j=1}^{t-1}\left(\overline{a_{j}} d_{t+s-j}-\overline{c_{j}} b_{t+s-j}\right) \frac{j s(s+1)}{t+s+1-j} \\
& \quad+\sum_{j=t}^{t+s-1}\left(\overline{a_{j}} d_{t+s-j}-\overline{c_{j}} b_{t+s-j}\right) \frac{t(t+1)(t+s-j)}{j+1}=0
\end{aligned}
$$

for every $s, t \geq 1$. Now put $t+s=n \geq 2$, then the above is changed to (19) and we finish the proof.

Proposition 14. Let $u, v \in L^{\infty} \cap b^{2}$. If $H_{u}$ commutes with $H_{v}$, then there exist constants $\alpha, \beta \in \mathbb{C}$ satisfying $|\alpha|+|\beta| \neq 0$ such that $\alpha u+\beta v$ is a constant.

Proof. Suppose $H_{u}$ commutes with $H_{v}$. By Lemma 13, we have the equation (19). Now if we can show that for each fixed $n \geq 2$, the determinant of $(n-1)^{2}$ matrix $\left(\alpha_{j, k}\right)$ is not zero, then we will get

$$
\overline{a_{k}} d_{n-k}-\overline{c_{k}} b_{n-k}=0, \quad 1 \leq k \leq n-1 .
$$

Therefore, we have

$$
\overline{a_{k}} d_{j}=\overline{c_{k}} b_{j}, \quad k, j \geq 1,
$$

which will approach our desired result.

In fact, we need to apply the elementary transformation to the matrix $\left(\alpha_{j, k}\right)$. Fix $k$ th column in the matrix, where $1 \leq k \leq n-2$. Note $\alpha_{j, k}=$ $\frac{n-k}{k+1}(j+1) j, \alpha_{j, k+1}=\frac{n-k-1}{k+2}(j+1) j$ when $j \leq k$ and $\alpha_{k+1, k}=\frac{(n-k-1)(n-k) k}{n+1-k}$, $\alpha_{k+1, k+1}=\frac{(n-k-1)(k+2)(k+1)}{k+2}$. Now we multiply $-\frac{(n-k-1)(k+1)}{(n-k)(k+2)}$ to every entry of the $k$ th column and then add each to corresponding one of $(k+1)$ th column, we can see that the entry of $(k+1)$ th column becomes zero in $j$ th $(1 \leq j \leq k)$ row and $\frac{2(k+1)(n-k-1)(n+1)}{(k+2)(n+1-k)}$ in the $(k+1)$ th row. Now we apply the above process $n-2$ times in the order of $k=n-2, n-3, \ldots, 2,1$, then the original matrix becomes the lower triangular matrix which is denoted by $\left(\beta_{j, k}\right)$, where

$$
\beta_{j, k}= \begin{cases}0, & j<k, \\ \frac{2 k(n-k)(n+1)}{(k+1)(n+2-k)}, & j=k,\end{cases}
$$


for $2 \leq k \leq n-1$ and $\beta_{1,1}=\alpha_{1,1}=n-1$. Therefore,

$$
\operatorname{det}\left(\alpha_{j, k}\right)=\operatorname{det}\left(\beta_{j, k}\right)=(n-1) \prod_{k=2}^{n-1} \frac{2 k(n-k)(n+1)}{(k+1)(n+2-k)} \neq 0,
$$

thus (21) holds.

It is easy to see that, from the equalities (21), only one of the following three cases will occur:

Case $1 . b_{j}=d_{j}=0$ for all $j \geq 1$, i.e., $u$ and $v$ are analytic functions;

Case 2. $a_{j}=c_{j}=0$ for all $j \geq 1$, i.e., $u$ and $v$ are co-analytic functions;

Case 3. $\alpha[u-u(0)]+\beta[v-v(0)]=0$ for some $\alpha, \beta \in \mathbb{C}$ and $|\alpha|+|\beta| \neq 0$.

Now we show both Case 1 and Case 2 are included in Case 3. Indeed, for Case $1, u=f$ and $v=h$, so

$$
\left\langle H_{f} H_{h} K_{z}, K_{w}\right\rangle=\left\langle P\left[U\left(f H_{h} K_{z}\right)\right], K_{w}\right\rangle=\left\langle h U f K_{z}, K_{w}\right\rangle,
$$

and similarly $\left\langle H_{h} H_{f} K_{z}, K_{w}\right\rangle=\left\langle f U h K_{z}, K_{w}\right\rangle$. Thus, $H_{f} H_{h}=H_{h} H_{f}$ implies $\left.P[h U f-f U h) K_{z}\right]=0$, i.e., $\widetilde{T}_{h U f-f U h}\left(K_{z}\right)=0$. So we get $\widetilde{T}_{h U f-f U h}=0$ and this implies $h U f-f U h=0$. If $f=0$ or $h=0$, we have done. If $h \neq 0$, then by $\frac{f}{h}=\frac{U f}{U h}$ we see easily that $\frac{f}{h}$ is a constant, i.e., $f=\lambda h$ for some $\lambda \in \mathbb{C}$, which is the special case of Case 3 . If $f \neq 0$, the argument is similar.

Same reasoning applies for Case 2. We complete the proof.

Note that the condition $\alpha u+\beta v$ being a constant in the above result is a sufficient and necessary condition for commutativity of two Toeplitz operators with harmonic symbols on $b^{2}[7]$, but it is not sufficient for the small Hankel operators case, as Theorem 2 has said.

Now we can prove our second main result.

Proof of Theorem 2. The sufficiency is easy to verify by (6). We now show the necessity. Suppose $H_{u}$ commutes with $H_{v}$, then (14) holds and by Proposition 14, we can assume without loss of generality that

$$
f-a_{0}=\lambda\left(h-c_{0}\right), \quad g=\bar{\lambda} k
$$

for some $\lambda \in \mathbb{C}$.

If $\lambda=0$, then $u=f+\bar{g}=a_{0}$, a constant. The case of $a_{0}=0$ is trivial. If $a_{0} \neq 0$, then $v=U v$ by (5), that is, $h-h(0)=k^{*}$, so $v=h+\bar{k}=h(0)+k^{*}+$ $U k^{*}$, which is the special case of (b).

In what follows, we assume $\lambda \neq 0$. Using (22) and direct calculations, (14) gives

$$
\left(\overline{a_{0}}-\bar{\lambda} \overline{c_{0}}\right)\left(k-h^{*}+\overline{c_{0}}\right)=0
$$

which implies $a_{0}=\lambda c_{0}$ or $h=k^{*}+c_{0}$. Combining this with Proposition 14, it is easy to see that $a_{0}=\lambda c_{0}$ will give Case (a) and $h=k^{*}+c_{0}$ will give Case (b), completing the proof.

We give some corollaries to Theorem 2 . 
Corollary 15. Let $u, v \in L^{\infty}$.

(a) If $u$ and $v$ are both analytic or co-analytic, then $H_{u} H_{v}=H_{v} H_{u}$ if and only if $\alpha u+\beta v=0$ for some $\alpha, \beta \in \mathbb{C}$ with $|\alpha|+|\beta| \neq 0$.

(b) If one of $u, v$ is analytic and another is co-analytic, then $H_{u} H_{v}=H_{v} H_{u}$ if and only if $u=0$ or $v=0$ or $u$ and $v$ are two constants.

Notice that the above results are different from the same case of commuting Toeplitz operators on the harmonic Bergman space obtained in [5] and [7].

For harmonic function $u$, recall that $U u=u$ if and only if $u=\varphi+U \varphi$ for some analytic function $\varphi$, so the following result is also clear.

CoROllary 16. Let $u \in L^{\infty} \cap b^{2}$, then $H_{u}$ is a normal operator if and only if one of the followings holds:

(a) $u^{*}=\lambda u$ for some $\lambda \in \mathbb{C}$;

(b) $U u=u$ and $[u-u(0)]^{*}=\lambda[u-u(0)]$ for some $\lambda \in \mathbb{C}$.

Note that if $u(z)=\sum a_{j} z^{j}+\sum b_{j} \bar{z}^{j}$, then $u^{*}=\lambda u$ means that there exists a constant $\alpha$ such that all $\alpha a_{j}$ and $\alpha b_{j}$ are real-valued. So the above result indicates that the case of the normal small Hankel operator is also different from the case of normal Toeplitz operator obtained in [5], [16].

\section{Product of two small Hankel operators being another small Hankel operator}

In this section, we will investigate the product problem of two small Hankel operators and prove Theorems 3 and 4 . It is remarked here that, for same problem of Toeplitz operators case, there is only partial result for the quasihomogenenous symbols [9], [10] and no any consequence for harmonic symbols.

Let $C$ be a conjugation on a separable Hilbert space $\mathcal{H}$, that is, $C^{2}=I$ and $\langle C \varphi, C \psi\rangle=\langle\psi, \varphi\rangle$ for $\varphi, \psi \in \mathcal{H}$. A bounded linear operator $T$ on $\mathcal{H}$ is said to be $C$-complex symmetric, if $C T C=T^{*}$. For more details about this type of operators, one is referred to [12]. The following lemma is obvious by the definition of complex symmetric operator.

Lemma 17. Let $A, B, T$ are all $C$-complex symmetric operator on $\mathcal{H}$. Then $A B=T$ if and only if $B A=T$. In particular, each case implies $A B=B A$.

The above lemma is a simple property for complex symmetric operator, but for our study about product problem of Toeplitz operators or small Hankel operators, it is very useful and even unexpected. Indeed, let $C f(z)=\bar{f}$, then $C$ is a conjugation on $b^{2}$ and $T_{\phi}$ is a $C$-complex symmetric operator, i.e., $C T_{\phi} C=T_{\phi}^{*}$, [4]. Now set $C_{1}=C U$, then $C_{1}$ is also a conjugation on $b^{2}$ and

$$
C_{1} H_{\phi} C_{1}=C U H_{\phi} C U=C U U T_{\phi} C U=C T_{\phi} C U=T_{\phi}^{*} U=H_{\phi}^{*},
$$

which implies that $H_{\phi}$ is a $C_{1}$-complex symmetric operator. Applying the above lemma, we have 
Proposition 18. Let $\varphi, \psi, \phi \in L^{\infty}$. Then

(a) $T_{\varphi} T_{\psi}=T_{\phi}$ if and only if $T_{\psi} T_{\varphi}=T_{\phi}$, in this case $T_{\varphi} T_{\psi}=T_{\psi} T_{\varphi}$.

(b) $H_{\varphi} H_{\psi}=H_{\phi}$ if and only if $H_{\psi} H_{\varphi}=H_{\phi}$, in this case $H_{\varphi} H_{\psi}=H_{\psi} H_{\varphi}$.

So when we consider the product problem, we can use the results of commutativity obtained before. Note that in [9] the authors only proved the case of Toeplitz operators with homonegeneous symbols, but here the result is valid even for general symbols.

In order to prove Theorems 3 and 4, we also need the following lemma. Recall that Toeplitz operator $\widetilde{T}_{\phi}$ and small Hankel operator $\widetilde{H}_{\phi}$ on the Bergman space $L_{a}^{2}$ are defined by $\widetilde{T}_{\phi}(F)=P(\varphi F)$ and $\widetilde{H}_{\phi} F=P U(\phi F)$ for $F \in L_{a}^{2}$. For analytic function $\varphi$, it is easy to see $\widetilde{T}_{\psi} \widetilde{T}_{\varphi}=\widetilde{T}_{\varphi \psi}$ and $\widetilde{T}_{\bar{\varphi}} \widetilde{T}_{\psi}=\widetilde{T}_{\bar{\varphi} \psi}$.

Lemma 19. Let $\phi, \psi \in L^{\infty}$. Then $H_{\phi}=T_{\psi}$ if and only if $\phi=\psi=0$.

Proof. If $H_{\phi}=T_{\psi}$, then $P H_{\phi} P=P T_{\psi} P$, that is, $\widetilde{H}_{\phi}=\widetilde{T}_{\psi}$. It is known that a bounded linear operator $T$ on $L_{a}^{2}$ is a small Hankel operator if and only if $\widetilde{T}_{\bar{z}} T=T \widetilde{T}_{z}$ [11]. So $\widetilde{T}_{\bar{z}} \widetilde{T}_{\psi}=\widetilde{T}_{\psi} \widetilde{T}_{z}$, that is, $\widetilde{T}_{\bar{z} \psi}=\widetilde{T}_{z \psi}$, therefore $\bar{z} \psi=z \psi$, which means $\psi=0$. Hence, $\phi=0$ because of (3).

The converse is obvious and the proof is complete.

Now we proceed to prove Theorem 3 .

Proof of Theorem 3. It is obvious that (c) implies (a) and (b). For the direction of (a) or (b) implies (c), by Proposition 18 and Theorem 1, we only need to consider the following cases which will induce the case (c) of the theorem.

Case 1. $\varphi=0$. In this case, we see $H_{\phi}=0$, so $\phi=0$.

Case 2. $H_{e^{i p \theta} \varphi} H_{e^{i p \theta} \psi}=H_{\phi}$, where $\psi$ is a radial function. Suppose $\phi=$ $\sum_{k \in \mathbb{Z}} e^{i k \theta} \phi_{k}$, where each $\phi_{k}$ is a radial function. For every positive integers $m$ and $n$, by Lemma 8 and Remark 9 we have

$$
\left\langle H_{e^{i p \theta} \varphi} H_{e^{i p \theta} \psi} z^{m}, \overline{z^{n}}\right\rangle=0
$$

and

$$
\left\langle H_{\phi} z^{m}, \overline{z^{n}}\right\rangle=\left\langle H_{e^{i(n-m)} \phi_{n-m}} z^{m}, \overline{z^{n}}\right\rangle=2(n+1) \widehat{\phi_{n-m}}(m+n+2)\left\langle\overline{z^{n}}, \overline{z^{n}}\right\rangle .
$$

Hence, for integers $m, n>0, \widehat{\phi_{n-m}}(m+n+2)=0$. Let $j=n-m$, then $j \in \mathbb{Z}$ and $\widehat{\phi_{-j}}(2 m+j+2)=0$ for all positive integer $m$. Therefore, by Remark 6 we obtain $\phi_{-j}=0$ for each $j \in \mathbb{Z}$. So $\phi=0$ and $H_{e^{i p \theta} \varphi} H_{e^{i p \theta} \psi}=0$. It follows from Lemma 8 again that when $m>|p|$,

$0=H_{e^{i p \theta} \varphi} H_{e^{i p \theta} \psi}\left(z^{m}\right)=4(m+p+1)(m+1) \widehat{\varphi}(2 m+p+2) \widehat{\psi}(2 m+p+2) z^{m}$, which implies $\widehat{\varphi}(2 m+p+2) \widehat{\psi}(2 m+p+2)=0$ for all $m>|p|$. So $\varphi=0$ or $\psi=0$ by Remark 6 . 
Case 3. $H_{c} H_{\psi}=H_{\phi}$, where $c$ is a nonzero constant and $U \psi=\psi$. That is, $T_{c \psi}=H_{\phi}$, which gives $\psi=\phi=0$ by Lemma 19 , completing the proof.

The following result about the semi-commutativity of two small Hankel operators is an immediate consequence of Theorem 3.

Corollary 20. Let $p \in \mathbb{Z}, \varphi \in L^{\infty}$ be radial and $\psi \in L^{\infty}$. Then the following statements are equivalent:

(a) $H_{e^{i p \theta} \varphi} H_{\psi}=H_{e^{i p \theta} \varphi \psi}$;

(b) $H_{\psi} H_{e^{i p \theta} \varphi}=H_{e^{i p \theta} \varphi \psi}$;

(c) $\varphi=0$ or $\psi=0$.

Another consequence of Theorem 3 is about the zero product problem of two small Hankel operator which has independent interest. It can deduce the same conclusion for the Toeplitz operator since $T_{\phi} T_{\psi}=U\left(H_{\phi} H_{U \psi}\right) U$ by (2).

Corollary 21. Let $p \in \mathbb{Z}, \varphi \in L^{\infty}$ be radial and $\psi \in L^{\infty}$. Then

(a) $H_{e^{i p \theta} \varphi} H_{\psi}=0$, if and only if $H_{\psi} H_{e^{i p \theta} \varphi}=0$, if and only if $\varphi=0$ or $\psi=0$

(b) $T_{e^{i p \theta} \varphi} T_{\psi}=0$, if and only if $T_{\psi} T_{e^{i p \theta} \varphi}=0$, if and only if $\varphi=0$ or $\psi=0$.

We now prepare to prove Theorem 4. Using Lemma 11 and similar argument as done in (16), it follows from $H_{u} H_{v}=H_{\phi}$ that

$$
\begin{array}{cc}
A: & {\left[\widetilde{H}_{u}-1 \otimes P(\bar{u})\right]\left[\widetilde{H}_{v}-1 \otimes P(\bar{v})\right]+\left[\widetilde{T}_{U u}-1 \otimes P\left(u^{*}\right)\right] \widetilde{T}_{v}} \\
& =\widetilde{H}_{\phi}-1 \otimes P(\bar{\phi}), \\
B: \quad & {\left[\widetilde{H}_{u}-1 \otimes P(\bar{u})\right]\left[\widetilde{T}_{U v}-1 \otimes P\left(v^{*}\right)\right]+\left[\widetilde{T}_{U u}-1 \otimes P\left(u^{*}\right)\right] \widetilde{H}_{U v}} \\
& =\widetilde{T}_{U \phi}-1 \otimes P\left(\phi^{*}\right), \\
C: & \widetilde{T}_{u}\left[\widetilde{H}_{v}-1 \otimes P(\bar{v})\right]+\widetilde{H}_{U u} \widetilde{T}_{v}=\widetilde{T}_{\phi}, \\
D: & \widetilde{T}_{u}\left[\widetilde{T}_{U v}-1 \otimes P\left(v^{*}\right)\right]+\widetilde{H}_{U u} \widetilde{T}_{U v}=\widetilde{H}_{U \phi} .
\end{array}
$$

The above four equalities will play great role for us to prove Theorem 4 .

We first give the following lemma.

Lemma 22. Let $u, v \in L^{\infty} \cap b^{2}, \phi \in L^{\infty}$. If $H_{u} H_{v}=H_{\phi}$, then $P(U \phi)=$ $P(\phi)$.

Proof. In view of Proposition 18, we see that $u$ and $v$ satisfy case (a) or case (b) of Theorem 2.

For case (a): If $\alpha=0$ or $\beta=0$, then $v=0$ or $u=0$, so we have $\phi=0$ which certainly implies $P(U \phi)=P(\phi)$.

For case (b): If $\alpha=0$, then we have $H_{\lambda_{1}} H_{\beta(\varphi+U \varphi)+\lambda_{2}}=H_{\phi}$, that is, by (2),

$$
T_{\lambda_{1}\left[\beta(\varphi+U \varphi)+\lambda_{2}\right]}=H_{\phi},
$$


then by Lemma 19 we have $\phi=0$ and $\lambda_{1} \beta \varphi=\lambda_{2}=0$. So $\varphi=0$ gives $P(U \phi)=$ $P(\phi)$ and $\lambda_{1} \beta \varphi=\lambda_{2}=0$ gives $u=0$ if $\lambda_{1}=0$ or $v=0$ if $\lambda_{1} \neq 0$. If $\beta=0$, the argument is similar.

Now in what follows, we only need to consider $\alpha \beta \neq 0$ in case (a) and (b) of Theorem 2. Without loss of generality, we may assume $\alpha=\beta=1$. So we let $u=h+\bar{k}+\lambda_{1}$ and $v=h+\bar{k}+\lambda_{2}$ with $h(0)=k(0)=0$, where $\lambda_{1}=\lambda_{2}$ or $h=k^{*}$.

We compute $C^{*}$ acting on 1 to obtain

$$
P(\bar{\phi})=P\left(k U k+\bar{h} h^{*}\right)+k h^{*}+\bar{\lambda}_{1} k+\bar{\lambda}_{2} h^{*}+\bar{\lambda}_{1} \bar{\lambda}_{2} .
$$

Because $H_{v^{*}} H_{u^{*}}=H_{\phi^{*}}$, so in this case we compute $D$ acting on 1 to obtain

$$
P\left(\phi^{*}\right)=P\left(k U k+\bar{h} h^{*}\right)+k h^{*}+\bar{\lambda}_{1} h^{*}+\bar{\lambda}_{2} k+\bar{\lambda}_{1} \bar{\lambda}_{2} .
$$

Since $\lambda_{1}=\lambda_{2}$ or $h=k^{*}$, then equations (23) and (24) give $P(\bar{\phi})=P\left(\phi^{*}\right)$, i.e., $P(U \phi)=P(\phi)$, completing the proof.

From the arguments of the above proof, we have seen that in order to prove Theorem 4, we only have to consider the case $H_{u} H_{v}=H_{\phi}$ when $u=h+\bar{k}+\lambda_{1}$ and $v=h+\bar{k}+\lambda_{2}$ with $h(0)=k(0)=0$, where $\lambda_{1}=\lambda_{2}$ or $h=k^{*}$. In this case, by straight calculations, $A$ and $B$ become

$$
\begin{aligned}
A: \quad \widetilde{H}_{\phi}- & 1 \otimes P(\bar{\phi}) \\
= & \widetilde{T}_{k^{*}+U h+\lambda_{1}} \widetilde{T}_{\bar{k}+h+\lambda_{2}}+\widetilde{H}_{\bar{k}} \widetilde{H}_{\bar{k}}-k^{*} \otimes k \\
& -1 \otimes\left[P\left(k U k+\bar{h} h^{*}\right)+k h^{*}+\bar{\lambda}_{1} k+\bar{\lambda}_{2} h^{*}+\bar{\lambda}_{1} \bar{\lambda}_{2}\right], \\
B: \quad \widetilde{T}_{U \phi}-1 \otimes P\left(\phi^{*}\right) & \\
= & \widetilde{H}_{\bar{k}} \widetilde{T}_{k^{*}}+\widetilde{T}_{U h+\lambda_{1}} \widetilde{H}_{U k+\lambda_{2}}+\widetilde{H}_{\bar{k}} \widetilde{T}_{U h+\lambda_{2}}+\widetilde{T}_{k^{*}} \widetilde{H}_{U h}-k^{*} \otimes h^{*} \\
& -1 \otimes\left[P\left(k U k+\bar{h} h^{*}\right)+k h^{*}+\bar{\lambda}_{1} h^{*}+\bar{\lambda}_{2} k+\bar{\lambda}_{1} \bar{\lambda}_{2}\right] .
\end{aligned}
$$

Using (23), (24) and (13), we can simplify $A$ and $B$ as

$A: \quad \widetilde{H}_{\phi}=\widetilde{T}_{k^{*}+U h+\lambda_{1}} \widetilde{T}_{\bar{k}+h+\lambda_{2}}+\widetilde{H}_{\bar{k}} \widetilde{H}_{\bar{k}}-k^{*} \otimes k$,

$B: \quad \widetilde{T}_{U \phi}=\widetilde{H}_{\bar{k} k^{*}+\left(h+\lambda_{1}\right)\left(U h+\lambda_{2}\right)}+\widetilde{H}_{\bar{k}} \widetilde{T}_{U h+\lambda_{2}}+\widetilde{T}_{k^{*}} \widetilde{H}_{U h+\lambda_{1}}-k^{*} \otimes\left(h+\lambda_{1}\right)^{*}$.

Also, $C$ and $D$ become

$C: \quad \widetilde{T}_{\phi}=\widetilde{H}_{\bar{k} k^{*}+\left(U h+\lambda_{1}\right)\left(h+\lambda_{2}\right)}+\widetilde{H}_{U h+\lambda_{1}} \widetilde{T}_{\bar{k}}+\widetilde{T}_{h+\lambda_{1}} \widetilde{H}_{\bar{k}}-\left(h+\lambda_{1}\right) \otimes k$,

$D: \quad \widetilde{H}_{U \phi}=\widetilde{T}_{\left(h+\lambda_{1}\right) k^{*}+\bar{k} k^{*}+\left(U h+\lambda_{2}\right) \bar{k}}$

$$
+\widetilde{T}_{h+\lambda_{1}} \widetilde{T}_{U h+\lambda_{2}}+\widetilde{H}_{U h+\lambda_{1}} \widetilde{H}_{U h+\lambda_{2}}-\left(h+\lambda_{1}\right) \otimes\left(h+\lambda_{2}\right)^{*} .
$$

Note that $B, D$ are defined on $L_{a}^{2}$ and $A, C$ are defined on $z L_{a}^{2}$. It is easy to see $A$ and $C$ also hold when acting on 1 because of (23), (24) and $P(U \phi)=[P(\bar{\phi})]^{*}$ or $P(\phi)=\left[P\left(\phi^{*}\right)\right]^{*}$. So the above $A, B, C, D$ are well defined on $L_{a}^{2}$.

We now turn to prove Theorem 4. 
Proof of Theorem 4. It is easy to see that we only need to show (a) implies (c). Since $\phi$ is a harmonic function, so by Lemma 22 we get $U \phi=\phi$. Let $\phi=\varphi+U \varphi+\lambda$, where $\varphi(z)=\sum_{j=1}^{\infty} a_{j} z^{j}$ is an analytic function on $\mathbb{D}$. By the previous arguments, we only need to show the theorem in the following case: $u=h+\bar{k}+\lambda_{1}$ and $v=h+\bar{k}+\lambda_{2}$, where $h(z)=\sum_{j=1}^{\infty} c_{j} z^{j}, k(z)=\sum_{j=1}^{\infty} d_{j} z^{j}$ and either $\lambda_{1}=\lambda_{2}$ or $h=k^{*}$.

Acting $C$ on $K_{\bar{z}}$, we get

$$
\begin{aligned}
{[\varphi+\varphi(z)+\lambda] K_{\bar{z}}=} & P\left[\left(k^{*} \bar{k}+\left(h+\lambda_{1}\right)\left(U h+\lambda_{2}\right)\right) \overline{K_{z}}\right] \\
& +k^{*}(z) P\left[\left(h+\lambda_{1}\right) \overline{K_{z}}\right]+\left(h+\lambda_{1}\right) P\left(k^{*} \overline{K_{z}}\right) \\
& -k^{*}(z)\left(h+\lambda_{1}\right) .
\end{aligned}
$$

Put $z=0$ in the above, and note $k(0)=\varphi(0)=0$, then we have

$$
P\left(k^{*} \bar{k}+h U h\right)=\varphi+\lambda-\left(h+\lambda_{1}\right) k^{*}-\lambda_{2} h-\lambda_{1} \lambda_{2} .
$$

Since $P\left[\overline{K_{z}}(I-P)\left(k^{*} \bar{k}+h U h\right)\right]=0$, then

$$
\begin{aligned}
P\left[\left(k^{*} \bar{k}+h U h\right) \overline{K_{z}}\right] & =P\left[\overline{K_{z}} P\left(k^{*} \bar{k}+h U h\right)\right] \\
& =P\left[(\varphi+\lambda) \overline{K_{z}}\right]-P\left[\left(h+\lambda_{1}\right) k^{*} \overline{K_{z}}\right]-\lambda P\left(h \overline{K_{z}}\right)-\lambda_{1} \lambda_{2} .
\end{aligned}
$$

Insert the above into (25), we thus get

$$
\begin{aligned}
& h P\left(k^{*} \overline{K_{z}}\right)+k^{*}(z) P\left(h \overline{K_{z}}\right)-P\left(h k^{*} \overline{K_{z}}\right)-k^{*}(z) h \\
& \quad=[\varphi+\varphi(z)+\lambda] K_{\bar{z}}-P\left[(\varphi+\lambda) \overline{K_{z}}\right] .
\end{aligned}
$$

Note that the left-hand side of the above is similar to each one side of (15), so it gives a similar formula as (20), that is, for each $t \geq 1$,

$$
\begin{aligned}
& \sum_{s=0}^{\infty} \frac{-1}{t+s+1}\left[\sum_{j=1}^{t} c_{j} \overline{d_{t+s-j}} \frac{j s(s+1)}{t+s+1-j}\right. \\
& \left.\quad+\sum_{j=t+1}^{t+s-1} c_{j} \overline{d_{t+s-j}} \frac{t(t+1)(t+s-j)}{j+1}\right] z^{s} .
\end{aligned}
$$

Note $K_{\bar{z}}(w)=\sum_{j=0}^{\infty}(j+1) z^{j} w^{j}$, then using (18) and straight computations, the right-hand side of the equality (26) gives that for each $t \geq 0$,

$$
\sum_{s=0}^{t}(s+1) a_{t-s} z^{s}+\sum_{s=t+1}^{\infty}(t+1) a_{s-t} z^{s}-\sum_{s=0}^{\infty} \frac{(t+1)(s+1)}{t+s+1} a_{t+s} z^{s},
$$

where $a_{0}=\lambda$. Comparing the coefficient of $z^{s}$ in the above two formulas which are equal from (26), we then get for $1 \leq s \leq t$,

$$
\begin{aligned}
& \sum_{j=1}^{t} c_{j} \overline{d_{t+s-j}} \frac{j}{t+s+1-j}+\sum_{j=t+1}^{t+s-1} c_{j} \overline{d_{t+s-j}} \frac{t(t+1)(t+s-j)}{s(s+1)(j+1)} \\
& \quad=\frac{t+1}{s} a_{s+t}-\frac{t+s+1}{s} a_{t-s} .
\end{aligned}
$$


Claim: $a_{n}=0$ for all nonnegative integer $n$.

In fact, let $t=s+n$ in the above equality, where $n \geq 0$, then the equality (27) becomes

$$
\begin{aligned}
& \sum_{j=1}^{s+n} c_{j} \overline{d_{2 s+n-j}} \frac{j}{2 s+n+1-j} \\
& \quad+\frac{(s+n)(s+n+1)}{s(s+1)} \sum_{j=s+n+1}^{2 s+n-1} c_{j} \overline{d_{2 s+n-j}} \frac{2 s+n-j}{j+1} \\
& \quad=\frac{s+n+1}{s} a_{2 s+n}-\frac{2 s+n+1}{s} a_{n}
\end{aligned}
$$

for all $s \geq 1$. Note

$$
\left|\sum_{j=1}^{s+n} c_{j} \overline{d_{2 s+n-j}} \frac{j}{2 s+n+1-j}\right| \leq \sum_{j=1}^{s+n}\left|c_{j} d_{2 s+n-j}\right|
$$

and

$$
\left|\sum_{j=s+n+1}^{2 s+n-1} c_{j} \overline{d_{2 s+n-j}} \frac{2 s+n-j}{j+1}\right| \leq \sum_{j=s+n+1}^{2 s+n-1}\left|c_{j} d_{2 s+n-j}\right|=\sum_{j=1}^{s-1}\left|d_{j} c_{2 s+n-j}\right|,
$$

then it is easy to see that, for each fixed nonnegative integer $n$, by (28), the claim will follow if we show

$$
E_{s}=\sum_{j=1}^{s+n}\left|c_{j} d_{2 s+n-j}\right| \rightarrow 0, \quad F_{s}=\sum_{j=1}^{s-1}\left|d_{j} c_{2 s+n-j}\right| \rightarrow 0
$$

as $s \rightarrow \infty$. Indeed, note $u=h+\bar{k}+\lambda_{1}$ is bounded on $\mathbb{D}$, so $h$ and $k$ are both in the Hardy space $H^{2}$, which means $\sum_{j}\left|c_{j}\right|^{2}<\infty$ and $\sum_{j}\left|d_{j}\right|^{2}<\infty$. Thus for any $\varepsilon>0$, there exists an integer $N>0$ such that $\sum_{j=N+1}^{\infty}\left|c_{j}\right|^{2}<\frac{\varepsilon}{2}$ and $\sum_{j=N+1}^{\infty}\left|d_{j}\right|^{2}<\frac{\varepsilon}{2}$. It is easy to see that there exists $M>N$ such that when $s>M,\left|d_{s}\right|<\varepsilon / 2\left(\left|c_{1}\right|+\cdots+\left|c_{N}\right|\right)$. Notice that $2 s+n-j \geq s$ when positive integer $j \leq s+n$. Thus, using the Hölder's inequality we have

$$
\begin{aligned}
E_{s} & =\sum_{j=1}^{N}\left|c_{j} d_{2 s+n-j}\right|+\sum_{j=N+1}^{s+n}\left|c_{j} d_{2 s+n-j}\right| \\
& <\sum_{j=1}^{N}\left|c_{j}\right|\left|d_{2 s+n-j}\right|+\left(\sum_{j=N+1}^{s+n}\left|c_{j}\right|^{2}\right)^{\frac{1}{2}}\left(\sum_{j=N+1}^{s+n}\left|d_{2 s+n-j}\right|^{2}\right)^{\frac{1}{2}} \\
& <\sum_{j=1}^{N}\left|c_{j}\right| \frac{\varepsilon}{2\left(\left|c_{1}\right|+\cdots+\left|c_{N}\right|\right)}+\left(\frac{\varepsilon}{2}\right)^{\frac{1}{2}}\left(\frac{\varepsilon}{2}\right)^{\frac{1}{2}}=\varepsilon
\end{aligned}
$$

as $s>M$, which means $E_{s} \rightarrow 0$ as $s \rightarrow \infty$. Same argument shows $F_{s} \rightarrow 0$ as $s \rightarrow \infty$, thus (29) holds and the claim is true. 
The claim means that $\phi=0$. Hence, (26) becomes

$$
h P\left(k^{*} \overline{K_{z}}\right)+k^{*}(z) P\left(h \overline{K_{z}}\right)-P\left(h k^{*} \overline{K_{z}}\right)-k^{*}(z) h=0 .
$$

Just as the arguments in the proof of Proposition 14, the above will give

$$
c_{k} \overline{d_{j}}=0, \quad j, k \geq 1,
$$

which implies either $h=0$ or $k=0$.

Case 1: $\phi=h=0$. Put this condition into $D$, we get $\widetilde{T}_{k^{*} \bar{k}+\lambda_{1} \lambda_{2}+\lambda_{1} k^{*}+\lambda_{2} \bar{k}}=$ 0 , which means $k^{*} \bar{k}+\lambda_{1} \lambda_{2}+\lambda_{1} k^{*}+\lambda_{2} \bar{k}=0$, or $k^{*} \bar{k}=-\lambda_{1} \lambda_{2}-\lambda_{1} k^{*}-\lambda_{2} \bar{k}$. So $k^{*} \bar{k}$ is harmonic on $\mathbb{D}$, which easily gives $k=0$ since $k(0)=0$ and also $\lambda_{1} \lambda_{2}=0$. Therefore, we get either $u=h+\bar{k}+\lambda_{1}=0$ if $\lambda_{1}=0$ or $v=h+\bar{k}+$ $\lambda_{2}=0$ if $\lambda_{2}=0$, as desired.

Case 2: $\phi=k=0$. Put this condition into $A$, we get $\widetilde{T}_{\left(U h+\lambda_{1}\right)\left(h+\lambda_{2}\right)}=0$, so $\left(U h+\lambda_{1}\right)\left(h+\lambda_{2}\right)=0$ which gives $h U h=-\lambda_{1} \lambda_{2}-\lambda_{1} h-\lambda_{2} U h$, so similarly $h=0$ and $\lambda_{1} \lambda_{2}=0$. Arguments as the Case 1 give the desired conclusion.

The proof is complete.

Now we have a solution to the zero product problem of two small Hankel operators as well as two Toeplitz operator with harmonic symbols on the harmonic Bergman space, just as in Corollary 21.

Corollary 23. Let $u, v \in L^{\infty} \cap b^{2}$. Then $H_{u} H_{v}=0$ if and only if $u=0$ or $v=0 ; T_{u} T_{v}=0$ if and only if $u=0$ or $v=0$.

The next rephrasing of Corollary 23 is a cancellation law for small Hankel operators or Toeplitz operators.

Corollary 24. Let $u, v_{1}, v_{2} \in L^{\infty} \cap b^{2}$ and $u$ is not identically 0. Then $H_{u} H_{v_{1}}=H_{u} H_{v_{2}}$ implies $v_{1}=v_{2}$ and $T_{u} T_{v_{1}}=T_{u} T_{v_{2}}$ implies $v_{1}=v_{2}$.

The next corollary says there are no nonzero idempotent small Hankel operators with bounded harmonic symbols.

Corollary 25. If $u$ is bounded and harmonic and $H_{u}^{2}=H_{u}$, then $u=0$.

For semi-commutativity of small Hankel operators, we have the following consequence.

Corollary 26. Let $u, v \in L^{\infty} \cap b^{2}$ and one of them is analytic or coanalytic. Then the following statements are equivalent:

(a) $H_{u} H_{v}=H_{u v}$;

(b) $H_{v} H_{u}=H_{u v}$;

(c) $u=0$ or $v=0$.

Proof. Suppose $u$ is analytic on $\mathbb{D}$. By Proposition 18 and Theorem 2, we have one of the following cases to discuss. 
Case 1: $\alpha u+\beta v=0$ for some $|\alpha|+|\beta| \neq 0$. In this case, if $\alpha=0$, then $v=0$; if $\beta=0$, then $u=0$; if $\alpha \beta \neq 0$, note $u$ is analytic, then $v$ is also analytic, so $u v$ is analytic, hence by Theorem 4 we obtain $u=0$ or $v=0$.

Case 2: $u=\alpha(\varphi+U \varphi)+\lambda_{1}$ and $v=\beta(\varphi+U \varphi)+\lambda_{2}$ for some analytic function $\varphi$ with $\varphi(0)=0$. In this case, since $u$ is analytic, so $\varphi=0$ or $\alpha \beta=0$, both cases will deduce $u$ or $v$ is a constant. Therefore, by (2) and Lemma 19, we get $u=0$ or $v=0$.

For the remaining cases the argument is similar. We finish the proof.

Acknowledgments. This work was partly done during the first and the second authors visiting the Department of Mathematics and Statistics, State University of New York at Albany, in the year 2014. They wish to express their great gratitude to the institution for hospitality and Professors Kehe Zhu and Rongwei Yang for helpful discussions. They also would like to thank CSC for providing living stipend in Albany and referee for his patience and useful comments.

\section{REFERENCES}

[1] P. Ahern and Z̆. Cuučković, A theorem of Brown-Halmos type for Bergman space Toeplitz operators, J. Funct. Anal. 187 (2001), 200-210. MR 1867348

[2] S. Axler and Z̆. Čučković, Commuting Toeplitz operators with harmonic symbols, Integral Equations Operator Theory 14 (1991), 1-12. MR 1079815

[3] A. Brown and P. R. Halmos, Algebraic properties of Toeplitz operator, J. Reine Angew. Math. 213 (1964), 89-102. MR 0160136

[4] Y. Chen, H. Koo and Y. J. Lee, Ranks of complex skew symmetric operators and its applications to Toeplitz operators, J. Math. Anal. Appl. 425 (2015), 734-747. MR 3303888

[5] B. Choe and Y. J. Lee, Commuting Toeplitz operators on the harmonic Bergman spaces, Michigan Math. J. 46 (1999), 163-174. MR 1682896

[6] Z̆. Čučković and N. V. Rao, Mellin transform, monomial symbols, and commuting Toeplitz operators, J. Funct. Anal. 154 (1998), 195-214. MR 1616532

[7] X. Ding, A question of Toeplitz operators on the harmonic Bergman space, J. Math. Anal. Appl. 344 (2008), no. 1, 367-372. MR 2416312

[8] X. T. Dong and Z. H. Zhou, Commuting quasihomogeneous Toeplitz operators on the harmonic Bergman space, Complex Anal. Oper. Theory 7 (2013), no. 4, 1267-1285. MR 3079855

[9] X. T. Dong and Z. H. Zhou, Product equivalence of quasihomogeneous Toeplitz operators on the harmonic Bergman space, Studia Math. 219 (2013), 163-175. MR 3149557

[10] X. T. Dong and Z. H. Zhou, Products of Toeplitz operators on the harmonic Bergman space, Proc. Amer. Math. Soc. 138 (2010), no. 5, 1765-1773. MR 2587461

[11] N. S. Faour, A theorem of Nehari type, Illinois J. Math. 35 (1991), 533-535. MR 1115985

[12] S. R. Garcia and M. Putinar, Complex symmetric operators and applications, Trans. Amer. Math. Soc. 358 (2006), 1285-1315. MR 2187654

[13] K. Guo and D. Zheng, Essentially commuting Hankel and Toeplitz operators, J. Funct. Anal. 201 (2003), 121-147. MR 1986157

[14] K. Guo and D. Zheng, Toeplitz algebra and Hankel algebra on the harmonic Bergman space, J. Math. Anal. Appl. 276 (2002), 213-230. MR 1944347 
[15] K. Guo and D. Zheng, Invariant subspaces, quasi-invariant subspaces, and Hankel operators, J. Funct. Anal. 187 (2001), no. 2, 308-342. MR 1875150

[16] Y. J. Lee and K. Zhu, Some differential and integral equations with applications to Toeplitz operators, Integral Equations Operator Theory 44 (2002), 466-479. MR 1942036

[17] I. Louhichi, E. Strouse and L. Zakariasy, Products of Toeplitz operators on the Bergman space, Integral Equations Operator Theory 54 (2006), 525-539. MR 2222982

[18] I. Louhichi and L. Zakariasy, Quasihomogeneous Toeplitz operators on the harmonic Bergman space, Arch. Math. (Basel) 98 (2012), no. 1, 49-60. MR 2885532

[19] I. Louhichi and L. Zakariasy, On Toeplitz operators with quasihomogeneous symbols, Arch. Math. (Basel) 85 (2005), 248-257. MR 2172383

[20] Y. Lu and B. Zhang, Commuting Hankel operator and Toeplitz operator on the Bergman space, Chinese Ann. Math. Ser. A 32 (2011), no. 5, 519-530. MR 2907202

[21] R. Martinez-Avendano, When do Toeplitz and Hankel operators commute? Integral Equations Operator Theory 37 (2000), 341-349. MR 1776959

[22] R. Remmert, Classical topics in complex function theory, Graduate Texts in Methematics, vol. 172, Springer, New York, 1998. MR 1483074

[23] K. Zhu, Duality and Hankel operators on the Bergman spaces of bounded symmetric domains, J. Funct. Anal. 81 (1988), 260-278. MR 0971880

Yong Chen, Department of Mathematics, Zhejiang Normal University, Jinhua, Zhejiang, 321004, P.R. China

E-mail address: ychen227@gmail.com; ychen@zjnu.cn

Wei He, Department of Mathematics, Southeast University, Nanjing, Jiangsu, 211189, P.R. CHINA

E-mail address: hewei.seu@gmail.com

Yunzhong Hu, Department of Mathematics, Zhejiang Normal University, Jinhua, Zhejiang, 321004, P.R. China

E-mail address: 21hyz@163.com 